\title{
Cholesteatoma and Otosclerosis: Two slowly progressive causes of hearing loss treatable through corrective surgery
}

James J. Holt, MD, Department of Otolaryngology-Head and Neck Surgery, Marshfield Clinic, Marshfield, Wisconsin

\section{REPRINT REQUESTS:}

James J. Holt, MD

Department of Otolaryngology-

Head and Neck Surgery

Marshfield Clinic

1000 North Oak Avenue

Marshfield, WI 54449

Telephone: $715-387-5436$

Fax: 715-389-3808

Email: holt.james@marshfieldclinic.org

\section{KEYWORDS:}

Ear diseases; Ear, middle; Cholesteatoma, Otorhinolaryngologic diseases;

Otosclerosis, Stapedectomy

\section{RECEIVED:}

MARCH 17, 2003

REVISED AND ACCEPTED:

MARCH 20, 2003

Clinical Medicine \& Research

Volume 1, Number 2: 151-154

(02003 Clinical Medicine \& Research

www.mfldclin.edu/clinmedres

\section{CHOLESTEATOMA}

Cholesteatoma is a unique disease of the ear in which a skin cyst grows into the middle ear and mastoid. The cyst is not cancerous but can erode tissue and cause destruction of the ear (figure 1).

There are several theories on how a cholesteatoma forms. Most evidence indicates that improper function of the Eustachian tube contributes to the formation of a cholesteatoma. If the Eustachian tube does not open often enough to equalize the pressures in the middle ear, negative pressure will develop behind the ear drum. This causes the drum to become retracted, forming a pocket. As the pocket deepens, it becomes trapped in the ear as a skin cyst or sac. Like skin tissue any-

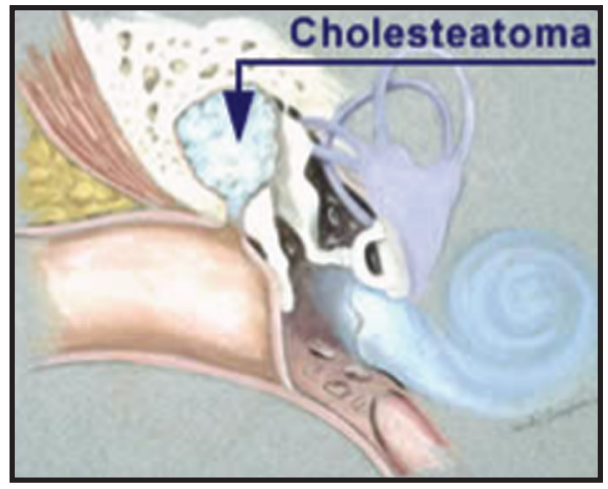

Figure 1. where in the body, dead skin cells slough off. This also occurs in the cholesteatoma sac. As more dead skin cells slough off, the sac gradually expands and a cholesteatoma develops. In other cases, skin grows around the margin of a perforation into the middle ear.

Patients who develop a cholesteatoma commonly have had previous problems with middle ear fluid and/or infections. However, it may be years before the cholesteatoma forms. It is also important to understand that most patients with a history of ear fluid/infections do not develop a cholesteatoma.

Infections in the ear are common with cholesteatoma and can lead to a foul smelling discharge that may contain blood. Antibiotics, either systemic (by mouth) or as ear drops, can help control the infection, but will not cure the patient of the cholesteatoma.

Most of the time, patients do not know a cholesteatoma is present. The expanding sac generally causes destruction of the ear drum and ossicles (bones of hearing). This causes hearing loss. There can be discharge from the ear. It is usually intermittent (comes and goes), but can be persistent. Sometimes this discharge is bloody. 
Cholesteatoma is not often painful. However, infection may occasionally occur, causing pain and swelling behind the ear. A cholesteatoma is detected only by examining the ear and finding the disease. However, the physician may suspect the disease when some or all of the following are present:

Gradual loss of hearing

Discharge from the ear

- History of past infection/fluid

problems in the ear

In almost all cases, surgery is necessary to remove the disease. Occasionally, enough of the cholesteatoma debris can be removed in the office with periodic cleaning of the ear to control the disease. However, this approach is generally used in patients who cannot undergo surgery.

The cholesteatoma will usually grow or expand if not removed. With this growth, there is further destruction of the ear structures. The patient is at risk for further infections, which in some cases, can be quite severe (such as mastoiditis or meningitis). However, it is generally not necessary to remove this disease on an urgent basis.

To have an understanding of this type of surgery, some terms should be defined:

- Tympanoplasty: Surgery that involves the tympanum (middle ear). The tympanum is the area of the ear behind the ear drum where the bones of hearing (ossicles) are located.

- Mastoidectomy: Surgery performed in the mastoid, where disease may occur.

- Tympanomastoidectomy: A surgical procedure that involves both the tympanum and mastoid.

- Ossiculoplasty (ossicular reconstruction): Repair or reconstruction of the ossicles (bones of hearing). There are many techniques and many types of prostheses that can be used for the reconstruction.

There are two basic surgical approaches to the ear:

- Transcanal: Performed through the ear canal.

- Postauricular: Performed by making an incision behind the ear and moving the ear forward to allow exposure of the mastoid and middle ear.

There are a number of factors that are taken into account when the surgeon decides what procedure is best. These factors include:

The extent of the cholesteatoma

$\checkmark$ The size of the mastoid

The primary goal of the surgery is to remove the disease; the secondary goal is to restore or maintain hearing. The approach used depends partly upon the location and size of the cholesteatoma, and partly upon the preference and experience of the surgeon. In some cases, the approaches are combined to obtain the best exposure.
Any surgical procedure carries potential risks. These risks need to be discussed with the patient and/or family prior to surgery.

- Hearing Loss: There is a slight chance of hearing loss in the inner ear. This loss can be complete and permanent.

- Dizziness: Some people experience dizziness that resolves within a day of surgery. It is not likely for dizziness to be a persistent problem.

- Facial Paralysis: The nerve that innervates the muscles of the face courses through the ear. Therefore, there is a slight chance of a facial paralysis. This facial paralysis affects the movement of the facial muscles for closing of the eye, making a smile and raising the forehead. The paralysis could be partial or complete. It may occur immediately after surgery or have a delayed onset. Recovery can be complete or partial.

- Tinnitus (noises in the ear): Tinnitus can occur with surgery, but is an uncommon postoperative problem.

- Taste Abnormalities: A small nerve that innervates some of the taste and salivary function courses through the ear. After ear surgery, some patients experience an abnormal taste in the mouth or some dryness of the mouth. Many times this problem will improve over time.

Along with these otologic risks, any surgery carries the risk of anesthesia, bleeding, infection and other more remote operative problems. Most surgeries for cholesteatoma are performed under general anesthesia. Some can be performed with intravenous sedation and local anesthesia. Few are performed with local anesthesia only.

It is generally felt that the occurrence of these potential complications is less with surgeons who are well trained in otologic surgery, have experience in performing these surgeries and perform these procedures on a regular basis.

\section{OTOSCLEROSIS}

Otosclerosis is a condition affecting the stapes bone. A normal stapes bone moves like a small piston; sounds cause the piston to vibrate. In otosclerosis, a callus of bone accumulates on the stapes creating a partial fixation. This limits the movement of the stapes bone, which results in hearing loss (figure 2).

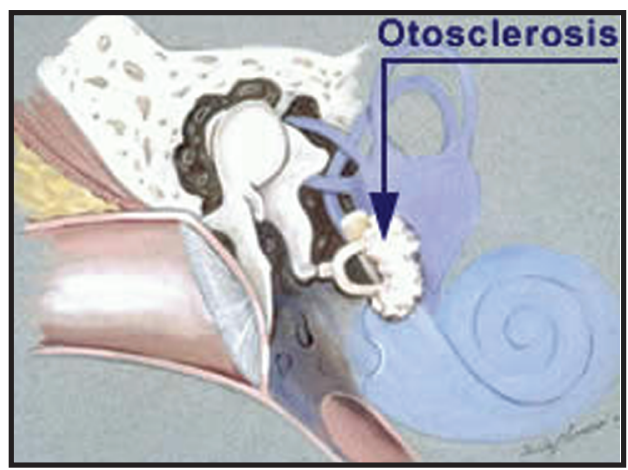

Figure 2.

$152 \quad C M \& R \quad 2003: 1$ (April) Holt


There is nothing a person does, or does not do to cause otosclerosis. There is a specific gene, which if present in the patient's genetic make up, can result in the development of otosclerosis. About $50 \%$ of patients with otosclerosis have a family history of otosclerosis. Most people do not have the gene that causes otosclerosis. Some medical studies have implicated the measles virus as a factor in causing otosclerosis.

Hearing loss is the most common symptom in patients with otosclerosis. Commonly, the hearing loss is noticed during the patient's late teens or early adulthood. The hearing loss progresses slowly in most cases. Many patients with otosclerosis experience tinnitus or a sound in the affected ear. There are some patients who experience a rather rapidly progressive hearing loss with otosclerosis. Pregnancy can accelerate the otosclerosis process, thus accelerating hearing loss.

Generally, otosclerosis begins in one ear. About $60 \%$ of the time, the other ear may become involved. Many times the involvement of the second ear occurs months or years later. In most cases, the hearing loss associated with otosclerosis will worsen with time.

On examination, the ear canal and eardrum are normal. Otosclerosis cannot be seen on an office examination. The audiogram indicates a conductive type of hearing loss. A special type of testing, stapedial reflexes, indicates limited or no movement of the bones of hearing.

There are three general treatment options:

- Observe and Recheck: For some patients, the hearing loss may not present a major problem. Therefore, one option is observation. However, it is best to recheck the hearing some time in the future, perhaps in one year. Patients with a milder degree of hearing loss may elect this option.

- Hearing Aid: A hearing aid is a device that amplifies sounds. Although it does not correct the underlying problem of otosclerosis, it does give the patient a nonsurgical option. Some hearing instrument specialists will allow a trial time of the hearing aid for a nominal fee. This gives the patient a chance to try the hearing aid before proceeding with a full purchase. There are other considerations. Some patients are concerned about the cost, the appearance, and the upkeep of the hearing aid and prefer a surgical option.

- Surgery (Stapedectomy): A stapedectomy is a surgical procedure that involves either a total or partial removal of the stapes bone. A prosthesis is then used to restore function.

\section{STAPEDECTOMY}

A stapedectomy is sometimes performed in patients who have a congenital abnormality of the stapes or have sustained a fracture of the stapes from a traumatic incident. However, the most common indication for a stapedectomy is otosclerosis.
Patients with otosclerosis and significant hearing loss receive audiometric (hearing) tests, an ear examination, and a consultation with the ear surgeon to determine the appropriateness of a stapedectomy.

During a stapedectomy, an incision is made in the skin of the ear canal, the skin and eardrum are lifted to expose the stapes bone, and the stapes bone is removed. An incision is made above the ear and the graft tissue is removed. This tissue is used to cover the opening created by the stapes bone removal. A prosthesis is put in place where the stapes bone had been and the ear drum and skin of the ear canal are laid back in place. The ear canal is then packed. Surgery is performed in one ear at a time. If surgery in the second ear is needed, it can be performed 6 months or more after the initial surgery.

Generally, a stapedectomy is not a very painful operation. The surgery is performed on an outpatient basis and takes about one hour to perform. Stapes surgery can be safely performed in children (patients as young as five years of age have undergone a stapedectomy).

Medication may be needed for a few days after the surgery. It generally takes about four weeks for the ear to heal. By the fourth week, patients normally notice an improvement of the hearing. It is important not to undergo any heavy or strenuous activity during this healing time. Generally, by the next day, patients are up and about. After the first week, patients may resume lighter activities.

No two surgeons perform the surgery exactly the same way. Virtually all stapedectomies are performed through the ear canal. Some surgeons use a vein from the arm or hand in place of the tissue from above the ear to close the opening created by removal of the stapes. There is a variety of prostheses that can be used, including a stainless steel piston, a wire, or others. Some surgeons remove part of the stapes bone, others essentially all of the stapes bone. The author in most cases prefers the method that involves the removal of most or all of the stapes bone, and placement of a Robinson prosthesis in most cases. This prosthesis has been used since the mid 1960's. The results have been excellent. In many cases, the author uses a laser to aid in the removal of the stapes bone.

The prosthesis is designed to be permanent. On occasion, there may be a problem with the healing process and the prosthesis may need to be revised. Sometimes the first surgery is not beneficial and a revision stapedectomy is recommended. This is essentially a stapedectomy that is performed in an ear that has already had one stapes procedure.

\section{CONCLUSION}

Cholesteatoma and otosclerosis are two causes of progressive hearing loss less familiar to the general practitioner. Increased awareness of these entities, and knowledge that they are generally correctable in the hands of an experienced otological surgeon, should aid in their wider recognition and treatment. 


\section{ACKNOWLEDGMENTS}

The author thanks Shirley Thompson for her illustration of figure 1 and 2 .

\section{FURTHER READINGS}

Holt JJ. Ear diseases and treatments Web site. Available at: http://www.marshfieldclinic.org/earsurgery. Accessed March 17, 2003.

Lippy WH, Burkey JM, Fucci MJ, Schuring AG, Rizer FM. Stapedectomy in the elderly. Am J Otol. 1996 Nov;17(6):8318344.

Lippy WH, Burkey JM, Schuring AG, Rizer FM. Stapedectomy in patients with small air-bone gaps. Laryngoscope. 1997 Jul;107(7):919-22.

Lippy WH, Burkey JM, Schuring AG, Rizer FM. Short- and longterm results of stapedectomy in children. Laryngoscope. 1998 Apr;108(4 Pt 1):569-572.

Shea JJ Jr. A personal history of stapedectomy. Am J Otol. 1998 Sep;19(5 Suppl):S2-12.

Shea PF, Ge X, Shea JJ Jr. Stapedectomy for far-advanced otosclerosis. Am J Otol. 1999 Jul;20(4):425-429.

Robinson M. The Robinson stapes prosthesis: a 15-year study. Otolaryngol Head Neck Surg. 1979 Jan-Feb;87(1):60-5.

Shambaugh GE, Glasscock ME: Pathology and clinical course of inflammatory diseases of the middle ear. In: Surgery of the Ear. 3rd ed. WB Saunders Company; 1980: 191, 200-207.

Sheehy JL. Acquired cholesteatoma in adults. Otolaryngol Clin North Am. 1989 Oct;22(5):967-979.

Soldati D, Mudry A. Knowledge about cholesteatoma, from the first description to the modern histopathology. Otol Neurotol. 2001 Nov;22(6):723-730. 